\title{
Conectividad y el uso de herramientas digitales en el aprendizaje del idioma inglés en estudiantes universitarios en tiempos del covid-19
}

\section{Connectivity and use of digital tools in English language learning among university students in times of covid-19}

Leonardo Efraín Cabezas. ${ }^{1}$, María Eugenia Rodríguez Durán . ${ }^{2}$ \& Patricia Pilar Moyota Amaguaya. ${ }^{3}$ Recibido: 18-11-2020 / Revisado: 15-12-2020 /Aceptado: 13-01-2021/ Publicado: 05-02-2021

\begin{abstract}
.
DOI: $\underline{\text { https://doi.org/10.33262/concienciadigital.v4i1.1.1565 }}$

Introduction. The online modality has been implemented in times of COVID-19 for the development of academic activities which puts at risk the accessibility to educational environments due to the lack of digital tools and poor connectivity. Objective. To know the reality of connectivity and accessibility to digital tools of the students of the Escuela Politécnica de Chimborazo. Methodology. 136 students participated in a descriptive survey with a total of 13 questions divided into 4 categories: Place of connectivity, electronic device, connectivity efficiency and virtual platforms. Results. $35.29 \%$ of students belong to Riobamba, $86.76 \%$ of students stay at home to receive online classes, $61.76 \%$ use computers to connect to their online classes, $61.03 \%$ do not own more than one device to connect to classes, $68.03 \%$ need to share their electronic device at home, 91. 91\% would choose a computer as a digital tool to attend their online classes, $77.21 \%$ did not specifically contract an internet service for their online classes, $53.28 \%$ have CNT as their provider, $47.06 \%$ have a slow internet speed and $41.18 \%$ have a fast internet speed. The quality of the Internet in $52.21 \%$ negatively affects the development of online classes in the option "sometimes". As for the platforms, $93.38 \%$ agreed that the Microsoft Team Platform is intuitive and easy to use, $51.47 \%$ use the Virtual Classroom as a means of support and $90.44 \%$ prefer the Microsoft Teams Platform for the development of their classes. Conclusion. It was concluded that not all students enjoy the same conditions of connectivity and digital tools which are necessary for a full academic development in a completely virtual environment in times of Covid-19.
\end{abstract}

Keywords: Covid-19, Higher education, Connectivity, Digital tools

\footnotetext{
1 Escuela Superior Politécnica de Chimborazo, Facultad de Administración de Empresas. Riobamba, Ecuador. leonardoe.cabezas@espoch.edu.ec

2 Escuela Superior Politécnica de Chimborazo, Facultad de Administración de Empresas. Riobamba, Ecuador. mariae.rodriguez@espoch.edu.ec

${ }^{3}$ Escuela Superior Politécnica de Chimborazo, Facultad de Ingeniería. Riobamba, Ecuador. patricia.moyota@espoch.edu.ec
} 


\section{Resumen}

Introducción. La modalidad online ha sido implementada en tiempos de COVID-19 para el desarrollo de las actividades académicas lo que pone en riesgo a la accesibilidad a los entornos educativos debido a la falta de herramientas digitales y mala conectividad. Objetivo. Conocer la realidad de conectividad y accesibilidad a las herramientas digitales de los y las de la Escuela Politécnica de Chimborazo. Metodología: 136 estudiantes participaron en una encuesta descriptiva con un total de 13 interrogantes divididas en 4 categorías: Lugar de conectividad, dispositivo electrónico, eficiencia de conectividad y plataformas virtuales. Resultados: $35.29 \%$ de estudiantes pertenecen a Riobamba, un $86.76 \%$ de los estudiantes se mantienen en su hogar para recibir clases online, un $61.76 \%$ usa computadoras para conectarse a sus clases online, un $61.03 \%$ no poseen más de un dispositivo para conectarse a clases, un $68.03 \%$ necesitan compartir su dispositivo electrónico en su hogar, un $91.91 \%$ escogerían una computadora como herramienta digital para asistir a sus clases en línea, un $77.21 \%$ no contrataron específicamente un servicio de internet para sus clases online, un $53.28 \%$ tiene como proveedor a la compañía CNT, en un $47.06 \%$ la velocidad de internet es lenta y un $41.18 \%$ rápida. La calidad del internet en un $52.21 \%$ afecta de forma negativa en el desarrollo de las clases online en la opción "a veces". En cuanto a las plataformas un 93.38\% estuvo de acuerdo en que la Plataforma Microsoft Team es intuitiva y fácil manejo, un $51.47 \%$ usa el Aula virtual como medio de apoyo y un $90.44 \%$ prefiere la Plataforma Microsoft Teams para el desarrollo de sus clases. Conclusión. Se concluyó que no todos los estudiantes gozan de las mismas condiciones de conectividad y de herramientas digitales las cuales son necesarias para un desarrollo académico pleno en un entorno completamente virtual en tiempos de Covid-19.

Palabras claves: Covid-19, Educación superior, Conectividad, Herramientas digitales

\section{Introducción.}

En diciembre de 2019, un brote de neumonía de origen desconocido comienza en la Provincia de Hubei China, alarmando a las organizaciones de salud a nivel mundial por su fácil transmisión y su desconocido tratamiento. (Esakandari et al., 2020). Luego de numerosos estudios en principios de enero de 2020 se pudo comprobar que el síndrome respiratorio agudo grave coronavirus 2 (SARSCoV-2) fue identificado como causa de la enfermedad y se lo enmarco por la Organización Mundial de la Salud (OMS) como “Coronavirus-19” (COVID-19). (Pascarella et al., 2020). Este virus fue reconocido como el agente causal de la neumonía epidémica en la ciudad de Wuhan, el cual por su gran poder de contagio se esparció rápidamente en toda China provocando la muerte de miles de personas al igual que en toda Europa principalmente en Italia donde los servicios médicos públicos y privados colapsaron dejando en evidencia el alto índice de propagación del virus. (Esakandari et al., 2020; Pascarella et al., 2020).

Para un rápido diagnóstico y el control del alto índice de contagio y muerte que se presentaban en los primeros meses se llevó a cabo un aislamiento de los ciudadanos para evitar una saturación de 
los servicios de salud. Al realizar este aislamiento se permitió efectuar en estos pacientes procedimientos terapéuticos de acuerdo a los datos epidemiológicos y clínicos con los que se contaban en esos momentos. Gracias a la globalización, el incremento de viajes a nivel mundial, las multitudes y la interacción humana el riesgo de contagios en todo el mundo aumentó y no solamente en el epicentro de la enfermedad sino en sectores aledaños y lejanos a este. Por estos motivos se presentaron los primeros casos en el continente americano a pocos meses de lo sucedido en Europa, sorpresivamente Estados Unidos uno de los países con un poder económico, científico y médico mayor a los de otros pudo vivir en carne propia la propagación y destrucción de esta enfermedad. El 11 de marzo de 2020, finalmente se la caracterizó como pandemia, luego de la gripe española (H1N1), gripe asiática (H2N2), la gripe de Hong Kong (H3N2) y en 2009 gripe pandémica (H1N1) que han causado un estimado de 50 millones, 1.5 millones, 1 millón y 300,000 muertes respectivamente. (N. P. A. S. Johnson \& Mueller, 2002). Gripes estacionales tienen un largo historial entre los seres humanos en diferentes puntos de la historia como remarcamos anteriormente. Una enfermedad pandémica se refiere aquella que se esparce alrededor de una región hacia una gran cantidad de países del mundo entero en donde los servicios de salud entran en "jaque" por un mayor número de pacientes que saturan las unidades de salud. (Kain \& Fowler, 2019).

Los departamentos de emergencia como la Unidad de cuidados intensivos fueron, son y serán los encargados de recibir a esta clase de pacientes. Lamentablemente la infraestructura, el número de profesionales y medicamentos no abastecen de manera adecuada la atención a las personas con diferentes síntomas y signos de sospecha de COVID-19. De igual manera al ser una enfermedad recientemente desarrollada por los seres humanos no se cuentan con la data científica que correlacione la eficacia de una terapéutica médica con el ocaso de la enfermedad en una persona contagiada. (Esakandari et al., 2020; Pascarella et al., 2020).

Esta realidad se extrapola a países tercermundistas que además de las necesidades económicas, culturales y educativas que presentaban en su entorno antes de la llegada de la pandemia su presente se vio gravemente afectado por no contar con los medios económicos, de infraestructura y culturales que permitan llevar un adecuado control sobre este problema de salud mundial. Entre los países más afectados a nivel de Latinoamérica se encuentran Brasil, México y Ecuador en los cuales se evidenció una alta mortalidad en contraste al número de casos reportados como código COVID19. (Santana-Sardi et al., 2020). A la llegada del paciente "cero" al Ecuador, el 16 de marzo de 
2020 se emplean diferentes medidas por parte del Estado para evitar un contagio masivo, entre las cuales se destacaron restricciones de movilidad, aislamiento de personas no esenciales para la producción del estado y el cierre de negocios y cese de actividades. (Santana-Sardi et al., 2020). Las diferentes condiciones para un confinamiento forzoso, paralización de actividades y distanciamiento social hizo que la ciudadanía altere toda su agenda cotidiana por una "nueva realidad", la misma que se la percibía de carácter temporal, pero en tiempos actuales este pensamiento sería inapropiado dado los hechos que se han palpado diariamente desde que las medidas fueron acatadas.

Todas estas condiciones se aplicaron para el sector público y privado mermando de esta forma las acciones de cotidianidad que se practicaban diariamente, dichas condiciones afectaron a diferentes actores de la sociedad y su rol en la comunidad. Desde un punto de vista de obligatoriedad se ha condicionado de una forma inexorable el funcionamiento de diferentes instituciones de salud, educativas, económicas y sociales. Se podría analizar que la pandemia trae consigo crisis en instituciones de salud y económicas por el cierre de actividades que suponen un ingreso a las arcas del estado y a las empresas privadas.

La educación desde niveles primarios hasta superiores se ha visto afectados de forma inmediata, debido al cierre de instituciones que trabajan de manera presencial en distintos programas de educación. Millones de estudiantes tuvieron que parar sus actividades de forma forzosa, las mismas que se venían desarrollando en un contexto presencial para "optar" por otras herramientas que suponen la transición a una modalidad diferente. (Vivanco-Saraguro et al., 2020). Si bien esta modalidad no es para nada nueva en el Ecuador un alto porcentaje de estudiantes ha venido recibiendo clases en un entorno convencional teniendo como fuente auxiliar herramientas digitales, sin ser éstas el actor principal y responsable de su educación. Este hecho no es único en el Ecuador y se lo puede apreciar de forma global lo que supone un impacto significativo en la calidad de aprendizaje y en la forma de impartir clases por parte de los docentes.

El enfoque no está centrado solamente en el estudiante y maestro, sino en aquellos actores ajenos a las aulas que participan en los procesos sustantivos de la educación, llámense, académicos, administrativos, personal de apoyo y autoridades que ahora más que nunca han desarrollado nuevas estrategias para mitigar el impacto en la calidad y la accesibilidad de la educación a nivel superior. (Miguel, 2020). 
Estas "nuevas" estrategias están encaminadas a usar y aprovechar las cuantiosas herramientas digitales a favor de la educación, las que permitirán mejorar la experiencia del estudiante en la formación a distancia que se ha desarrollado de forma obligada durante esta pandemia. (Román, 2020). Estas estrategias están basadas en el empleo de las Tecnologías de la Información y las Comunicaciones (TICs) un campo el cual se modifica y evoluciona de una forma inimaginada gracias a los avances tecnológicos y sus aplicaciones, las mismas que permiten contraponerse a diferentes adversidades y se destacan por ser opciones fiables y de un carácter de alta y mediana accesibilidad. (García Bejarano et al., 2013).

Estas herramientas suponen una ruptura de las barreras del distanciamiento y tiempo, las que potencian logros y alcances, siempre y cuando estén fundamentadas en raíces pedagógicas que supongan un fortalecimiento de las buenas prácticas en la educación. (Herrera, 2015). Si bien es cierto todas estas herramientas virtuales se han usado como un recurso de apoyo a la educación presencial, los estudiantes que han venido acostumbrados a desarrollar sus actividades en un entorno convencional con la instalación de la pandemia se han visto obligados a usar estos recursos de apoyo como su principal medio de aprendizaje. Las nuevas tecnologías han apoyado en esta transición forzada, lo que ha sido una gran ventaja pero llama la atención es que, si nos quedamos con una herramienta de apoyo sin trabajar en un cambio total del aprendizaje en estos tiempos de pandemia y logramos que el estudiante pueda desarrollar las competencias necesarias para el uso adecuado del conocimiento que se imparte, se corre el riesgo de que en un futuro, no muy lejano, se repita un modelo de educación tradicional que no permita llegar a un mayor número de estudiantes. (Garcia Bejarano et al., 2013).

La sociedad es un producto de contextos y momentos, de sus actividades, las cuales son altamente dinámicas e interrelacionadas en un carácter profundo dado por la globalización y con la llegada del internet supuso un cambio total en la forma de comunicación en un mundo altamente tecnológico y competitivo. Como se mencionó la creación del internet abrió la posibilidad de un cambio de época, para muchos pudo ser el comienzo de TICs hay que enmarcar hechos memorables que ya distinguían estos tipos de tecnología. La invención del teléfono en 1876, la radio en 1898, el tubo de vacío en 1906, el primer ordenador en 1951 son claros ejemplos del uso de tecnología en la forma de comunicación en la sociedad. (Garcia Bejarano et al., 2013).

En consecuencia, de estos desarrollos como menciona (Garcia Bejarano et al., 2013), se encuentra 
que el cambio significativo está marcado por el campo de las comunicaciones y es el primer fenómeno a recalcar ya que la sobreabundancia de la información tiene una esencia y un propósito que cambia la percepción del mundo, el modo de pensar y de actuar. La educación convencional a tratado de gestionar y suministrar la información en donde el maestro "transmitía" su conocimiento a los estudiantes como ley absoluta. Esto fue útil durante mucho tiempo ya que era la única manera de acercarse a los conocimientos de otras personas, pero la realidad ha cambiado ya que al tener mejores canales de comunicación se puede encontrar información valiosa al alcance de un click. En consecuencia, desde el proceso formativo y los cambios que se han venido dando se espera que los estudiantes y todos los actores involucrados con la educación tengan las capacidades de buscar la información de forma crítica y responsable para la creación de conocimiento creativo, basado en evidencia y traspolarlo a un ambiente práctico. (Herrera, 2015).

La aparición de las TICs en la ecuación impacta de una manera directa a la educación presencial y a distancia, dichas en otras palabras se da lugar a una educación virtual la cual entra en un contexto de nuevos ambientes telemáticos que logran efectuar una comunicación efectiva entre seres humanos y específicamente entre alumno y maestro. (Paredes Chacín et al., 2020). Este nuevo contexto brinda la oportunidad de acceder a nueva información al instante que hace pocos años hubiera sido inimaginable, el mismo que puede ser compartido en tiempo real o en forma asincrónica. Estas ventajas suponen nuevos estilos de enseñanza - aprendizaje, y las estrategias metodológicas representan un gran potencial de poder ser diversificadas hacia una nueva demanda logrando ofertar un estilo de educación que se adecue al medio cambiante condicionado por la pandemia que cruzamos. La ventaja que puede suponer estas condiciones de enseñanza radica en la familiaridad que tienen las nuevas generaciones con la tecnología y su aplicación en la vida diaria. Con esto sale a relucir el término "nativo digital" propuesto por Marc Prensky, el que ha desarrollado y ha descrito como un fenómeno social en el que los jóvenes están "hiperconectados" que aprenden y que se relacionan de una forma muy natural a los medios tecnológicos en contraste con generaciones anteriores a los cuales el los enmarca como "inmigrantes digitales" (García et al., 2007).

Con esta metáfora se podrá ejemplificar claramente que los alumnos corresponden a todos estos nativos digitales y los maestros que en su mayoría no ha tenido un cambio generacional en inmigrantes digitales los que deben adaptarse en mayor proporción a esta realidad. Los nativos digitales tienen nuevas fortalezas derivadas de las capacidades tecnológicas las mismas que están 
ISSN: 2600-5859

dirigidas por herramientas digitales que usan a diario, pero también existen debilidades que pueden comprometer su desarrollo profesional y su futuro. Entre estas carencias están la falta de formación y disciplina intelectual que experimentan diferentes grupos y ponen en riesgo no solamente su futuro personal, sino que se corre el riesgo de que países se mantengan en la vanguardia en la producción de ciencia y tecnología. (Piscitelli, 2008). No hay duda de la importancia en las destrezas tecnológicas en la vida productiva, social y académica, pero ésta puede verse truncada con un bajo desarrollo de competencias analíticas y de producción intelectual. A este fenómeno, se lo denomina "ignorantes analógicos" (García et al., 2007). Con ello se pone a discusión el reto en educación, y se replantea el fortalecimiento de procesos críticos y de forma estratégica apoyados en TICs, con ello se permitirá crear actividades y proveer recursos a través de plataformas que sean amigables con las generaciones nativas en la información.

Es verdad que el ente de formación corresponde a los alumnos en el 50\% sin embargo no se puede dejar de lado a los maestros que cierran el otro 50\%. Si bien es cierto con el implemento de nuevas tecnologías el entorno de trabajo de los maestros a dado un giro de 180 grados, ya que estos deben acoplarse a un sistema del cual no han sido parte ni se han capacitados dejándoles más dudas que certezas. El rol del profesor en la educación superior debe adecuarse a todas estas innovaciones ya que de no ser así no podrán ofrecer una educación de calidad. Se deberá ajustar metodologías, capacitación para los docentes, creación de plataformas y mejoras a las ya constituidas con una correcta planificación donde haya una retroalimentación en todos los sectores de la educación. (Garcia Bejarano et al., 2013). El docente de la antigua escuela siempre debe estar abierto a la idea de adquirir nuevos conocimientos para poder producir aulas adecuadas y que el alumno pude alcanzar la cima del aprendizaje, por tanto, el rol del maestro será conectarse con el estudiante por medio de herramientas digitales las cuales son familiares para el aprendiz y su entorno.

Si bien hemos conversado acerca de la calidad y la accesibilidad de un entorno digital debemos también consolidar el pensamiento humanístico el cual dimensiona el valor de la dignidad humana en las relaciones personales. (Expósito \& Marsollier, 2020). Este eje procura que la educación debe estar centrada en los valores humanos y no solo en la asimilación del conocimiento, ya que la formación del ser es un ente complejo que abarca diferentes puntos de vista en donde la conservación y respeto de la diversidad social y cultural permiten un desarrollo pleno en la educación de la persona. (Vinueza \& Simbaña, 2017). Si conjugamos estas dos realidades que se han venido detallando podemos correlacionar estas dos temáticas que se han suscitado de manera 
individual ahora se han correlacionado en un mismo lapso de tiempo. Hablamos de la pandemia y la educación usando medios tecnológicos como piedra angular dados los acontecimientos a los cuales estamos expuestos. (Santana-Sardi et al., 2020).

Al hablar específicamente del aprendizaje del idioma inglés podemos percibir que los estudiantes en la actualidad tienen un entorno de enseñanza de carácter visual y auditivo el cual puede ser dirigido en gran medida por herramientas tecnológicas. El aprendizaje del idioma inglés como segunda lengua en países hispanohablantes ha tenido un cambio extraordinario ya que antes se lo concebía como un proceso privilegiado para un sector exclusivo ahora se lo encuentra en la mayoría de mallas curriculares en sector públicos y privados. (Cabezas et al., 2019). Este cambio a permitido percibir al aprendizaje del idioma inglés como un pilar fundamental para el progreso y la generación de nuevas oportunidades. Si bien antes suponía ser una ventaja en diferentes situaciones laborales y estudiantiles ahora es un requerimiento indispensable a la hora de superación personal y el alcanzar objetivos laborales individuales y colectivos.

Como es de conocimiento la innovación de cursos y estrategias de enseñanza virtual del idioma inglés como lengua extranjera por medio de plataformas digitales se han posicionado en el mercado como una alternativa a la realidad en el medio ecuatoriano en el que el estudiante prefiere una enseñanza tradicional con un contacto frente a frente con el maestro. (Cabezas et al., 2019). Sin duda este tipo de preferencias cambiará en el transcurso de la pandemia que vivimos ya que por ahora el estudiante universitario está obligado a cambiar el entorno de aprendizaje por uno que sea completamente online.

Muchas ventajas se pueden percibir al recibir este tipo de enseñanzas como la facilidad de traslado a clases ya que por medio de un dispositivo tecnológico el estará en clases sin la necesidad de abandonar su hogar, otra ventaja es que no deberá tener un presupuesto destinado para el transporte, comida y actividades extras en un formato educativo presencial. Si bien podemos apreciar que existen muchas ventajas acerca de este método de educación una serie de preguntas pueden entran en la discusión sobre el entorno educativo en tiempos de COVID-19: ¿Los alumnos tienen todas las herramientas digitales para recibir clases en un entorno $100 \%$ online? ¿Las plataformas virtuales brindan un acceso e interfaz intuitivo y fácil de manejar? ¿Los estudiantes poseen servicios de internet que garanticen un adecuado desarrollo de sus clases? ¿Todos los alumnos gozan de las mismas condiciones de accesibilidad que les garantice un aprendizaje igual al que uno convencional? Por este motivo el presente estudio tuvo como finalidad conocer la realidad de 
conectividad y accesibilidad a las herramientas digitales de los y las estudiantes que cursan el aprendizaje del idioma inglés como lengua extranjera de la Escuela Politécnica de Chimborazo, dado que no todos los estudiantes gozan con las mismas oportunidades en cuanto a los medios tecnológicos y a la accesibilidad para desarrollar todas sus aptitudes y actitudes en un entorno de aprendizaje $100 \%$ en línea.

\section{Metodologia.}

\section{Población de estudio}

Un total de 136 estudiantes de la Escuela Superior Politécnica de Chimborazo (ESPOCH) participaron en este estudio. Los estudiantes pertenecen a las carreras de Administración de Empresas y de Transporte los mismos que estaban matriculados en el 3er y 4to nivel de la asignatura de inglés en el período 2020-2021.

\section{Criterios de inclusión}

Estudiantes universitarios que pertenezcan a la ESPOCH que cursen el 3er y 4to nivel de la asignatura de inglés en el período 2020-2021 que pertenezcan a las carreras de Administración de Empresas y Transporte.

\section{Criterios de exclusión}

Estudiantes universitarios que no pertenezcan a la ESPOCH y que cursen un nivel diferente al 3er y 4to de la asignatura de inglés en el período 2020-2021 y que no pertenezcan a las carreras de Administración de Empresas y de Transporte.

\section{Diseño del estudio}

El modelo de estudio fue de carácter descriptivo, observacional y transversal la obtención de los datos se los realizó por medio de una encuesta descriptiva de preguntas cerradas y abiertas divididas en cuatro categorías con un total de 13 preguntas. La primera categoría está relacionada con el lugar de conectividad, la segunda a los dispositivos electrónicos usados para la conectividad en clases online, la tercera a la calidad de internet que los estudiantes usan para desempeñar sus actividades y la última a la plataforma virtual usada para el desarrollo de clases. Se empleó el software Microsoft Form que permite acceder a la encuesta por medio de un enlace URL el cual fue enviado a todos los estudiantes de las carreras mencionadas a través de la Plataforma Microsoft Teams en el desarrollo de las actividades de clase programadas en su horario habitual.

\section{Análisis Estadístico}

Se utilizó un análisis estadístico descriptivo empleando el software Excel con el mismo que se cuantificaron 
ISSN: 2600-5859

las respuestas en relación al número total de participantes, promedios de cada respuesta y porcentaje con su mayor o menor aparición en la selección de los estudiantes.

\section{Resultados.}

Los resultados y preguntas se detallan en las tablas 1-13.

Tabla1. Pregunta 1 ¿De qué ciudad usted se conecta para sus clases de inglés?

\begin{tabular}{lcc}
\hline Pregunta 1 & Cantidad & Porcentaje \\
\hline Riobamba & 48 & 35 \\
Ambato & 8 & 6 \\
Santo Domingo de los Tsáchilas & 7 & 5 \\
Guaranda & 6 & 4 \\
Quito & 5 & 4 \\
Cañar & 4 & 3 \\
Puyo & 4 & 3 \\
Guano & 4 & 3 \\
Baños de Agua Santa & 3 & 2 \\
Latacunga & 3 & 2 \\
Lago Agrio & 3 & 2 \\
Macas & 2 & 1 \\
Saquisili & 2 & 1 \\
Penipe & 2 & 1 \\
Ibarra & 2 & 1 \\
La Joya de los Sachas - Orellana & 2 & 1 \\
Tena & 2 & 1 \\
Otras Ciudades & 29 & 21 \\
Total & $\mathbf{1 3 6}$ & $\mathbf{1 0 0 \%}$ \\
\hline
\end{tabular}

Fuente: Plataforma Virtual

Elaborado por: Investigadores

Tabla 2. Pregunta 2 ¿Para recibir sus clases usted permanece en su hogar o necesita trasladarse a otro sitio de estudio?

\begin{tabular}{lcc}
\hline Pregunta 2 & Cantidad & Porcentaje \\
\hline $\begin{array}{l}\text { Hogar } \\
\text { Lugar ajeno a su residencia }\end{array}$ & 118 & 87 \\
(casa de familiar, café net) & 18 & 13 \\
Total & $\mathbf{1 3 6}$ & $\mathbf{1 0 0 \%}$ \\
\hline $\begin{array}{l}\text { Fuente: Plataforma Virtual } \\
\text { Elaborado por: Investigadores }\end{array}$ & &
\end{tabular}

Tabla 3. Pregunta 3 ¿Qué dispositivo electrónico usted utiliza para conectarse a clases de inglés?

\begin{tabular}{lcc}
\hline Pregunta 3 & Cantidad & Porcentaje \\
\hline Computadora & 84 & 62 \\
Smartphone & 52 & 38 \\
Total & 136 & $\mathbf{1 0 0 \%}$ \\
\hline Fuente: Plataforma Virtual & \\
Elaborado por: Investigadores &
\end{tabular}


ISSN: 2600-5859

Tabla 4. Pregunta 4 ¿Usted posee más de 1 dispositivo para conectarse a clases en forma virtual?

\begin{tabular}{lcc}
\hline Pregunta 4 & Cantidad & Porcentaje \\
\hline Sí & 53 & 39 \\
No & 83 & 61 \\
Total & $\mathbf{1 3 6}$ & $\mathbf{1 0 0 \%}$ \\
\hline
\end{tabular}

Fuente: Plataforma Virtual

Elaborado por: Investigadores

Tabla 5. Pregunta 5 ¿Usted se ve en la necesidad de compartir su dispositivo electrónico con más integrantes de su familia para el desarrollo de clases o trabajos en forma virtual?

\begin{tabular}{lcc}
\hline Pregunta 5 & Cantidad & Porcentaje \\
\hline Sí & 93 & 68 \\
No & 43 & 32 \\
Total & $\mathbf{1 3 6}$ & $\mathbf{1 0 0 \%}$ \\
\hline Fuente: Plataforma Virtual & \\
Elaborado por: Investigadores
\end{tabular}

Tabla 6. Pregunta 6 ¿Si usted pudiera elegir un dispositivo electrónico para recibir sus clases de inglés cual sería su elección?

\begin{tabular}{lcc}
\hline Pregunta 6 & Cantidad & Porcentaje \\
\hline Computadora & 125 & 92 \\
Smartphone & 11 & 8 \\
Total & 136 & $\mathbf{1 0 0 \%}$ \\
\hline Fuente: Plataforma Virtual & \\
Elaborado por: Investigadores &
\end{tabular}

Tabla 7. Pregunta 7 ¿Usted se ha visto obligado a contratar un servicio de internet fijo exclusivamente para el desarrollo de clases virtuales?

\begin{tabular}{lcc}
\hline Pregunta 7 & Cantidad & Porcentaje \\
\hline Sí & 105 & 77 \\
No & 31 & 23 \\
Total & $\mathbf{1 3 6}$ & $\mathbf{1 0 0 \%}$ \\
\hline Fuente: Plataforma Virtual & \\
Elaborado por: Investigadores
\end{tabular}

Tabla 8. Pregunta 8 ¿Qué proveedor de internet usted posee?

\begin{tabular}{lcc}
\hline Pregunta 8 & Cantidad & Porcentaje \\
\hline CNT & 73 & 54 \\
Otro & 45 & 33 \\
Netlife & 12 & 9 \\
Claro & 5 & 4 \\
Movistar & 1 & 1 \\
Total & $\mathbf{1 3 6}$ & $\mathbf{1 0 0 \%}$ \\
Fuente: Plataforma Virtual & \\
Elaborado por: Investigadores
\end{tabular}


ISSN: 2600-5859

Tabla 9. Pregunta 9 ¿Cómo describiría la velocidad de conexión de internet en sus clases de inglés?

\begin{tabular}{lcc}
\hline Pregunta 9 & Cantidad & Porcentaje \\
\hline Lenta & 64 & 47 \\
Normal & 56 & 41 \\
Muy lenta & 14 & 10 \\
Rápida & 2 & 2 \\
Total & $\mathbf{1 3 6}$ & $\mathbf{1 0 0 \%}$ \\
\hline
\end{tabular}

Fuente: Plataforma Virtual

Elaborado por: Investigadores

Tabla 10. Pregunta 10 ¿La calidad de conexión a internet influye de forma negativa en el desarrollo de sus clases de inglés?

\begin{tabular}{lcc}
\hline Pregunta 10 & Cantidad & Porcentaje \\
\hline A veces & 71 & 52 \\
Casi siempre & 42 & 31 \\
Siempre & 19 & 14 \\
Casi nunca & 4 & 3 \\
Total & $\mathbf{1 3 6}$ & $\mathbf{1 0 0 \%}$ \\
\hline Fuente: Plataforma Virtual & \\
Elaborado por: Investigadores &
\end{tabular}

Tabla 11. Pregunta 11 ¿La plataforma Microsoft Teams le ofrece una interfaz intuitiva y de fácil accesibilidad para sus clases de inglés?

\begin{tabular}{lcc}
\hline Pregunta 11 & Estudiantes & Porcentaje \\
\hline Sí & 127 & 93 \\
No & 9 & 7 \\
Total & $\mathbf{1 3 6}$ & $\mathbf{1 0 0 \%}$ \\
\hline
\end{tabular}

Fuente: Plataforma Virtual

Elaborado por: Investigadores

Tabla 12. Pregunta 12 ¿Además de Microsoft Teams a que otra plataforma usted accede para complementar su aprendizaje en el idioma inglés?

\begin{tabular}{lcc}
\hline Pregunta 12 & Estudiantes & Porcentaje \\
\hline $\begin{array}{l}\text { Aula virtual } \\
\text { Videos educativos en plataformas }\end{array}$ & 70 & 51 \\
como Youtube, Facebook, Instagram & 34 & 25 \\
o Tik - Tok & & \\
Aplicaciones inteligentes & 15 & 11 \\
Blogs & 12 & 9 \\
Otros & 5 & 4 \\
Total & $\mathbf{1 3 6}$ & $\mathbf{1 0 0 \%}$ \\
\hline
\end{tabular}

Fuente: Plataforma Virtual

Elaborado por: Investigadores 
Tabla 13. Pregunta 13 ¿Qué plataforma educativa usted preferiría para recibir clases?

\begin{tabular}{lcc}
\hline Pregunta 13 & Estudiantes & Porcentaje \\
\hline Microsoft Teams & 123 & 90 \\
Google Meet & 4 & 3 \\
Zoom & 7 & 5 \\
Otra & 2 & 2 \\
Total & $\mathbf{1 3 6}$ & $\mathbf{1 0 0 \%}$ \\
\hline Fuente: Plataforma Virtual & \\
Elaborado por: Investigadores &
\end{tabular}

\section{Discusión}

La pandemia ha tenido un impacto directo en las actividades cotidianas y educativas en la población mundial. Las restricciones de movilidad y el cierre de centros educativos han sido las medidas que más han afectado al Sistema de Educación Superior lo que ha condicionado que exista la apertura de modalidades online lo cual genera una posible brecha para los estudiantes y profesores que no cuentan con las herramientas digitales adecuadas para el desempeño de las actividades de enseñanza y aprendizaje. En tal virtud el objetivo de este estudio fue conocer la realidad de conectividad y accesibilidad a las herramientas digitales de los y las estudiantes que cursan el aprendizaje del idioma inglés como lengua extranjera de la Escuela Politécnica de Chimborazo. Las encuestas online se han realizado desde los años 90 del siglo XX, al tiempo en el que se popularizaron los cursos a distancia a través de plataformas del aprendizaje como WebCT, Moodle o Blackboard (Román, 2020). Las ventajas de las encuestas digitales son numerosas ya que son fáciles de crear y de distribuir, además los resultados se pueden analizar rápidamente y su implementación y acceso son más eficientes.

A pesar de tener numerosas ventajas existen limitaciones como la baja participación del alumnado ya que su colaboración se ve mermada por la no asistencia de los alumnos a sus clases y el sesgo de no respuesta; sin embargo en este estudio la encuesta fue direccionada a 140 estudiantes de los cuales 136 lo pudieron resolver en su totalidad brindado datos que se utilizaron para su análisis lo cual concuerda con los resultados de estudios de Johnson y Thorpe donde concluyen que el índice de participación de los estudiantes no depende del medio usado para la distribución de las encuestas(T. D. Johnson, 2003; Thorpe, 2002). No existe un estudio a nivel ecuatoriano que rescate información acerca de la accesibilidad, calidad y el uso de diferentes herramientas digitales en educación superior en tiempos de Covid-19 siendo este un estudio pionero en el campo. 
En cuanto a la primera categoría podemos verificar que un 35,39\% de los estudiantes se encuentran en el cantón Riobamba mientras que el restante recibe sus clases en diferentes ciudades y provincias. Esta cifra remarca que más del $50 \%$ no se encuentra en las cercanías geográficas de la ESPOCH lo cual verifica la oferta académica que esta institución de educación superior brinda a todo el país. En esta misma categoría se comprobó que un 13,24\% de los estudiantes deben trasladarse fuera de su hogar para poder conectarse a sus clases online. No se especifica la distancia, el tipo de transporte y el tiempo requerido lo cual puede ser un determinante a la hora de relacionarlo con la situación socio-económica y la calidad de conectividad al igual con el riesgo de contagio ante el Covid-19. Esto se ve reflejado en datos relacionados con la accesibilidad de los hogares al servicio de internet en donde solamente se alcanza un 56.4\% (Miguel, 2020) lo que supone una falta de condiciones para que los estudiantes puedan realizar sus actividades académicas.

En la tercera categoría se pudo verificar que el $61.76 \%$ de los estudiantes usaba una computadora como herramienta digital para acceder a sus clases online, que un $61.03 \%$ no contaba con más de un dispositivo para conectarse a sus clases y que un $68.38 \%$ se veía en la necesidad de compartir su dispositivo digital con otro familiar. La preferencia y el uso de una computadora se correlaciona con el tráfico web el cual está distribuido por un: $75.49 \%$ en computadoras, $23.44 \%$ en celulares y $1 \%$ en tablets (Marboleda., 2020). lo cual concuerda con los resultados de las encuestas en donde un dispositivo electrónico tipo tablet no figura como elección para ningún estudiante en sus preferencias para de recibir clases online. Aproximadamente un $89 \%$ de la población se encuentra conectado a través de un dispositivo móvil y que el $69 \%$ de la población son usuarios de internet. (Santana-Sardi et al., 2020).

Se puede verificar con cierto asombro que menos del 35\% no se ven en la necesidad de compartir su dispositivo electrónico a lo largo de la jornada. Esto puede deberse a que en tiempos de Covid19 el flujo de conectividad a través de dispositivos electrónicos ha aumentado no solo por los estudiantes de primer, segundo y tercer nivel sino también por trabajadores que se ven en la obligación de realizar sus actividades por la modalidad de teletrabajo. Esta hipótesis puede corroborarse con un incremento del 6.8\% de usuarios activos en redes sociales (Marboleda., 2020). Para la tercera categoría en donde se buscaba información acerca de la eficiencia de conectividad de acuerdo al sistema de internet usado se observó que un $77.21 \%$ de estudiantes no se vio en la necesidad de contratar un plan fijo de internet exclusivamente para su uso en clases on-line lo cual 
ISSN: 2600-5859

nos indica que la mayoría de los estudiantes contaban con este servicio antes de la pandemia.

Se reflejo que un 53.68\% usan el proveedor de internet CNT, que un $47.06 \%$ y un $41.18 \%$ destacan una velocidad de conexión lenta y normal respectivamente y que en un $52.21 \%$ la calidad de internet que poseen los estudiantes interfiere de forma negativa en sus clases online.

Todos estos datos no pueden relacionar que el servicio brindado por la compañía CNT sea el causante de la mala conectividad, pero si pueden aseverar que una mala conectividad tiene un impacto directo en la calidad de aprendizaje de los estudiantes ya que al no contar con un buen servicio de internet la conectividad se ve limitada.

Un dato que debe ser resaltado es que ningún estudiante escogió una velocidad "muy rápida" de conectividad al igual que no se escogió la opción "nunca" en que si la calidad de conectividad influía de forma negativa en sus clases online lo que nos demuestra que una adecuada conectividad permite un desarrollo adecuado de clases y que los servicios de internet pueden llegar a saturarse o a su vez el servicio brindado por las compañías es limitado.

Por último, la cuarta categoría en donde se engloba a las plataformas y su uso en clases online se verificó que un 93,3\% de estudiantes posicionan a la plataforma Microsoft Teams como un medio de aprendizaje intuitivo y de fácil manejo y que un $90.44 \%$ escogieron a la misma como su primera opción para recibir clases. Esto nos demuestra que la plataforma Microsoft Teams brinda herramientas de conectividad y de simplicidad para el estudiante al recibir sus clases online.

Por otro lado, el $51.47 \%$ escogieron al aula virtual como otra plataforma de educación seguido por videos educativos en redes sociales esto está relacionado a que la mayoría de las actividades y su entrega están asignadas en un aula virtual mientras que la plataforma Teams se utiliza como medio de clases directo.

El posicionamiento de las redes sociales en la última década ha permitido el desarrollo de nuevas alternativas de educación. Las redes sociales Facebook, Instagram, YouTube y Tik Tok son líderes en audiencia y conectividad lo que es aprovechado de forma positiva con la incursión de videos educativos que permiten al estudiante tener más alternativas en el desarrollo de su conocimiento. (Santana-Sardi et al., 2020).

Los resultados obtenidos en este estudio deben tomarse con cautela ya que la muestra encuestada fue limitada a un grupo en específico de estudiantes y los datos provienen de alumnos que han 
ISSN: 2600-5859

podido matricularse en este periodo y no se ha tomado en cuenta la relación en cantidad de estudiantes pre y post pandemia lo que contribuiría a dilucidar una brecha mucho mayor en cuanto a la accesibilidad de educación en tiempos de Covid-19 ya que por diferentes motivos los estudiantes se vieron en la necesidad de posponer sus estudios a corto y mediano plazo.

Se recomienda ampliar este estudio con una muestra mayor y la medición en los niveles de satisfacción y aprendizaje de los estudiantes en un entorno totalmente virtual.

Tabla 14. Encuesta aplicada a estudiantes con sus cuatro categorías

\begin{tabular}{|c|c|c|}
\hline & ¿De qué ciudad usted se conecta para sus clases de inglés? & R. Abierta \\
\hline $\begin{array}{l}\text { Lugar de } \\
\text { conectividad }\end{array}$ & $\begin{array}{l}\text { ¿Para recibir sus clases usted permanece en su hogar o necesita } \\
\text { trasladarse a otro sitio de estudio? }\end{array}$ & $\begin{array}{ll}\text { A. } & \text { Hogar } \\
\text { B. Lugar ajeno a su } \\
\text { residencia (casa de } \\
\text { familiar, café net) }\end{array}$ \\
\hline \multirow{4}{*}{$\begin{array}{l}\text { Dispositivo } \\
\text { Electrónico }\end{array}$} & $\begin{array}{l}\text { ¿Qué dispositivo electrónico usted utiliza para conectarse a } \\
\text { clases de inglés? }\end{array}$ & $\begin{array}{l}\text { A. Computadora } \\
\text { B. Tablet } \\
\text { C. Smartphone }\end{array}$ \\
\hline & $\begin{array}{l}\text { ¿Usted posee más de } 1 \text { dispositivo para conectarse a clases en } \\
\text { forma virtual? }\end{array}$ & $\begin{array}{ll}\text { A. } & \mathrm{Si} \\
\text { B. } & \text { No }\end{array}$ \\
\hline & $\begin{array}{l}\text { ¿Usted se ve en la necesidad de compartir su dispositivo } \\
\text { electrónico con más integrantes de su familia para el desarrollo } \\
\text { de clases o trabajos en forma virtual? }\end{array}$ & $\begin{array}{ll}\text { A. } & \mathrm{Si} \\
\text { B. No }\end{array}$ \\
\hline & $\begin{array}{l}\text { ¿Si usted pudiera elegir un dispositivo electrónico para recibir } \\
\text { sus clases de inglés cuál sería su elección? }\end{array}$ & $\begin{array}{l}\text { A. Computadora } \\
\text { B. Tablet } \\
\text { C. Smartphone }\end{array}$ \\
\hline & $\begin{array}{l}\text { ¿Usted se ha visto obligado a contratar un servicio de internet } \\
\text { fijo exclusivamente para el desarrollo de clases virtuales? }\end{array}$ & $\begin{array}{ll}\text { A. } & \mathrm{Si} \\
\text { B. } & \text { No }\end{array}$ \\
\hline & ¿Qué proveedor de internet usted posee? & $\begin{array}{l}\text { A. CNT } \\
\text { B. Claro } \\
\text { C. Netlife } \\
\text { D. Movistar } \\
\text { E. Otro }\end{array}$ \\
\hline Eficiencia de & $\begin{array}{l}\text { ¿Cómo describiría la velocidad de conexión en sus clases, } \\
\text { describir en el recuadro que proveedor utiliza? }\end{array}$ & $\begin{array}{l}\text { A. Muy lenta } \\
\text { B. Lenta } \\
\text { C. Normal } \\
\text { D. Rápida } \\
\text { E. Muy rápida }\end{array}$ \\
\hline
\end{tabular}


ISSN: 2600-5859

\begin{tabular}{|c|c|c|}
\hline Conectividad & $\begin{array}{l}\text { ¿La calidad de conexión a internet influye de forma negativa en } \\
\text { el desarrollo de sus clases de inglés? }\end{array}$ & $\begin{array}{l}\text { A. Siempre } \\
\text { B. Casi siempre } \\
\text { C. A veces } \\
\text { D. Casi nunca } \\
\text { E. Nunca }\end{array}$ \\
\hline \multirow{3}{*}{$\begin{array}{l}\text { Plataformas } \\
\text { virtuales }\end{array}$} & $\begin{array}{l}\text { ¿La plataforma Microsoft Teams le ofrece una interfaz intuitiva } \\
\text { y de fácil accesibilidad para sus clases de inglés? }\end{array}$ & $\begin{array}{ll}\text { A. } & \mathrm{Si} \\
\text { B. No }\end{array}$ \\
\hline & $\begin{array}{l}\text { ¿Además de Microsoft Teams a que otra plataforma usted } \\
\text { accede para complementar su aprendizaje en el idioma inglés? }\end{array}$ & $\begin{array}{l}\text { A. Blogs } \\
\text { B. Aplicaciones inteligentes } \\
\text { C. Videos educativos en } \\
\text { plataformas como } \\
\text { YouTube, Facebook, } \\
\text { Instagram o Tik - Tok } \\
\text { D. Aula Virtual } \\
\text { E. Otros }\end{array}$ \\
\hline & ¿Qué plataforma educativa usted preferiría para recibir clases? & $\begin{array}{l}\text { A. Microsoft Teams } \\
\text { B. Google Meet } \\
\text { C. Zoom } \\
\text { D. Otra }\end{array}$ \\
\hline
\end{tabular}

Fuente: Plataforma Virtual

Elaborado por: Investigadores

\section{Conclusiones.}

- Con las limitaciones de este estudio se puede concluir que no todos los estudiantes gozan de las mismas condiciones de conectividad al igual que no todos poseen las mismas herramientas digitales las cuales son necesarias para un desarrollo académico pleno en un entorno completamente virtual.

- Dichas limitaciones tienden a relacionarse con la percepción de la calidad del aprendizaje el mismo que puede ser interpretado como negativo para el estudiante creando insatisfacción y falta de interés.

- Por consiguiente, se recomienda se tome en cuenta esta brecha de accesibilidad y de oportunidades en el desarrollo académico de los estudiantes en tiempos de Covid-19

\section{Referencias bibliográficas.}

Cabezas, L. E., Lara Freire, M. L., \& Rojas Yumisaca, W. G. (2019). Análisis de la influencia de las tareas en línea en las calificaciones de los estudiantes del nivel A1+ de inglés. Ciencia Digital, 3(4.2), 55-72. https://doi.org/10.33262/cienciadigital.v3i4.2.1004

Esakandari, H., Nabi-afjadi, M., Fakkari-afjadi, J., Farahmandian, N., Miresmaeili, S., \& 
Bahreini, E. (2020). A comprehensive review of COVID-19 characteristics. 2, 1-10.

Expósito, C. D., \& Marsollier, R. G. (2020). Virtualidad y educación en tiempos de COVID-19.

Un estudio empírico en Argentina. Educación y Humanismo, 22(39), 1-22.

https://doi.org/10.17081/eduhum.22.39.4214

Garcia Bejarano, A., Angarita, J., \& Velandia Mesa, C. (2013). Implicaciones pedagógicas del uso de las TICs en la educación superior. Revista de Tecnología, 12(3), 36-56. https://doi.org/10.18270/rt.v12i3.1750

García, F., Portillo, J., Romo, J., \& Benito, M. (2007). Nativos digitales y modelos de aprendizaje. CEUR Workshop Proceedings, 318.

Herrera, A. M. (2015). Una mirada reflexiva sobre las TIC en educación superior. Revista Electronica de Investigacion Educativa, 17(1), 1-4.

Johnson, N. P. A. S., \& Mueller, J. (2002). Updating the accounts: global mortality of the 19181920 "Spanish" influenza pandemic. Bulletin of the History of Medicine, 76(1), 105-115. https://doi.org/10.1353/bhm.2002.0022

Johnson, T. D. (2003). Online Student Ratings: Will Students Respond? New Directions for Teaching and Learning, 2003(96), 49-59. https://doi.org/10.1002/tl.122

Kain, T., \& Fowler, R. (2019). Preparing intensive care for the next pandemic influenza. Critical Care, 23(1), 1-9. https://doi.org/10.1186/s13054-019-2616-1

Marboleda, A. (2020). Estadísticas digitales Recuperado de. https://www.hablemosdemarcas.com/estadisticas-digitales-ecuador-2020/

Miguel, J. (2020). La educación superior en tiempos de pandemia : una visión desde dentro del proceso formativo. RLEE Nueva Época, L(Número especial), 13-40.

Paredes Chacín, A. J., Inciarte González, A., \& Walles Peñaloza, D. (2020). Educación superior e investigación en Latinoamérica: Transición al uso de tecnologías digitales por Covid-19. Revista de Ciencias Sociales, ISSN-e 1315-9518, Vol. 26, No. 3, 2020, Págs. 98-117, 26(3), 98-117. https://orcid.org/0000-0002-3682-6061

Pascarella, G., Strumia, A., Piliego, C., Bruno, F., Del Buono, R., Costa, F., Scarlata, S., \& Agrò, F. E. (2020). COVID-19 diagnosis and management: a comprehensive review. Journal of Internal Medicine, 288(2), 192-206. https://doi.org/10.1111/joim.13091

Piscitelli, A. (2008). Nativos Digitales. Universidad de Lima - Contratexto, 0(16), 43-56. https://revistas.ulima.edu.pe/index.php/contratexto/article/view/782

Román, E. (2020). La evaluación del profesorado universitario en tiempos de pandemia : los sistemas online de gestión de encuestas de satisfacción estudiantil. 61-70.

Santana-Sardi, G. A., Castro-Coello, R. L., Gutiérrez-Santana, J. A., \& Verónica Cecilia Zambrano-Palacios. (2020). La Educación Superior ecuatoriana en tiempo de la pandemia del Covid-19. 6, 757-775.

Thorpe, S. (2002). Online Student Evaluation of Instruction: An Investigation of Non-Response Bias. 42nd Annual Forum of the Association for Institutional Research, 1-10. http://dev.cms.aa.ufl.edu/Data/Sites/4/media/evals/OnlineEvaluationsAndNonResponseBias.pdf

Vinueza, S., \& Simbaña, V. (2017). Impacto de las TIC en la Educación Superior en el Ecuador. Revista Publicando, 4(11), 355-368.

https://rmlconsultores.com/revista/index.php/crv/article/view/530\%0Ahttp://www.rmlconsul 
tores.com/revista/index.php/crv/article/view/30

Vivanco-Saraguro, A., Internacional, C., Cáceres-muñoz, J., Martín-sánchez, M., Mexican, T., Tosso, M. P., Casado, C. M., Quiroz, C., Nacional, U., De México, A., Álvarez, M., Juárez Pérez-Cea, J., Oña Cots, J. M. de, Lorena, M., \& Bonilla-Guachamín, J. A. (2020).

Desigualdades educativas y la brecha digital en tiempos de COVID-19. CienciAmérica, 9(2), 115-121. https://revistas.uam.es/riejs/article/view/12111 


\section{PARA CITAR EL ARTÍCULO INDEXADO.}

Cabezas, L. E., Rodríguez Durán , M. E., \& Moyota Amaguaya, P. P. (2021). Conectividad y el uso de herramientas digitales en el aprendizaje del idioma inglés en estudiantes universitarios en tiempos del covid-19. ConcienciaDigital, 4(1.1), 307-326. https://doi.org/10.33262/concienciadigital.v4i1.1.1565

\section{\Ciencia}

El artículo que se publica es de exclusiva responsabilidad de los autores y no necesariamente reflejan el pensamiento de la Revista Conciencia Digital.

El artículo queda en propiedad de la revista y, por tanto, su publicación parcial y/o total en otro medio tiene que ser autorizado por el director de la Revista Conciencia Digital.

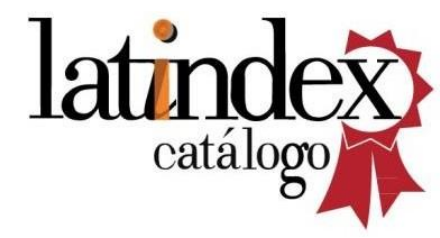

